\title{
Von der Schuldkultur zur Sicherheitskultur. Eine begriffsgeschichtliche Analyse 1986 - 2010
}

\author{
Valentin Rauer*
}

\begin{abstract}
The term "security culture" ("Sicherheitskultur") was first used in the public sphere of the Federal Republic of Germany in an inquiry report on the Chernobyl reactor accident. Since then, the frequency of use of the term has been increasing in print media. Compared to the period 2000-2004 the occurrence of the term almost doubled in the period 2005-2009. This process entails the disassociation of the concept from its original context and the emergence of new meanings and references. Among these, this analysis identifies two main semantic functions: a comparative function and an operational function. The comparative function is used to compare collective actors with respect to their approach to security issues. The operational function focuses on relations: the human-machine, human-human and human-rules interrelation. The latter draws attention not so much on rational risk calculations or wrongful actions, but on cultural contexts and interpretations. This reflects the transformation from a "culture of blame" to one of "safety culture" and the transition from an "asymmetrical" to a "symmetrical anthropology".
\end{abstract}

Keywords: Security, risk, nuclear incident, Chernobyl, actor-network theory, medicine Sicherheit, Risiko, Atomunfall, Tschernobyl, Akteur-Netzwerk-Theorie, Medizin

\section{Einleitung}

S obald sich auf der Welt ein schwerer Unfall ereignet, der die Gesundheit der Bevölkerung beeinträchtigen könnte, wird in den politischen Öffentlichkeiten über die Sicherheit der angewandten Technik diskutiert. Der Begriff der „Sicherheit“ hat dabei eine entscheidende Legitimationsfunktion. Wie die Antwort nach der Legitimität ausfällt, entscheidet sich daran, inwiefern Techniken als „sicher" oder „unsicher“ ausgewiesen werden. Die inhaltlichen Entgrenzungen von Sicherheitssemantiken breiten sich im Laufe der letzten Jahrzehnte stets weiter aus, und zwar gilt dies sowohl für Energietechnologien und Umweltrisiken als auch für kriegerische, terroristische oder gesundheitliche Gefahren (Daase 2010). „Sicherheit“ und „Sicherheitskulturen“ mögen in Expertenkreisen eindeutig definiert sein, in der Öffentlichkeit erfahren diese Konzepte wie alle anderen abstrakten Konzepte auch stets Bedeutungstransformationen und besitzen Eigenlogiken. Die Eigenlogiken des Konzepts „Sicherheitskultur" sollen in diesem Beitrag für die Jahre 1986-2010 nachgezeichnet werden. Historische Studien zum allgemeinen Sicherheitsbegriff und zu Sicherheitskonzepten liegen zwar vor, allerdings erfassen diese nicht die letzten zwanzig Jahre. Zudem fehlt bisher eine spezifische Begriffsgeschichte der "Sicherheitskultur“. ${ }^{1}$

Das Konzept „Sicherheitskultur“ wird seit dem Reaktorunfall von Tschernobyl im Jahre 1986 verwendet (Büttner/Fahlbruch/ Wilpert 1999). Seither, so zeigt die hier vorgestellte Analyse, erlebt das Konzept nicht nur eine stetige Bedeutungszunahme und erfährt einen quantitativen Sprung ab dem Jahr 2005. Vielmehr artikuliert es auch eigenlogische Semantiken. Semantisch lassen sich seit seiner Einführung zwei Hauptfunktionen iden-

* Valentin Rauer ist wissenschaftlicher Mitarbeiter im Forschungsprojekt „Sicherheitskultur im Wandel“, Goethe-Universität Frankfurt a. M., Kontakt: vrauer@soz.uni-frankfurt.de. Der Autor bedankt sich bei den anonymen Gutachtern bzw. Gutachterinnen für die sehr konstruktiven Kritiken und Hinweise.

1 Vgl. zur Geschichte des Sicherheitsbegriffs: Conze 1984; Conze 2005 sowie allgemein die Beiträge in: Zwierlein/Graf/Ressel 2010, dort insbesondere Daase 2010; Härter 2010; Schenk 2010. tifizieren: eine komparative und eine operative. Die komparative Funktion dient dazu, kollektive Akteure mit Blick auf ihren Umgang mit Sicherheit zu vergleichen, beispielsweise wenn es heißt, die „östliche Sicherheitskultur“ sei „weniger entwickelt“ als die „westliche“ etc. Ein solcher Vergleich relativiert und subjektiviert Sicherheitsfragen. Die operative Funktion nimmt hingegen Beziehungsverhältnisse in den Blick, beispielsweise wie Menschen mit Maschinen interagieren, wie sie Teamarbeit kultivieren oder welche Regelwerke sie wie befolgen etc. Die operative Funktion fragt also nach den Beziehungsverhältnissen zwischen Mensch-Maschinen, Mensch-Menschen und Mensch-Regeln. Laut Bruno Latour (1998) ging die Moderne bei diesen Beziehungsverhältnissen von einer asymmetrischen Anthropologie aus, die irrtümlich den Menschen entweder über die Technik oder die Technik über den Menschen stellte. Stattdessen ist dieses Verhältnis jedoch durch ein komplexes Akteur-Aktanten-Netzwerk charakterisiert, in dem der Mensch lediglich in Relation zu anderen Aktanten stehe (ebd.). Vor diesem Hintergrund konstituiert das Konzept der Sicherheitskultur die neue Position einer Re-Symmetrisierung des Beziehungsverhältnisses zwischen Mensch und Technik.

Methodisch wird dieser Weg des Konzepts mit einer an der historischen Semantik orientierten Analyse nachgezeichnet. Historische Semantik lenkt den Blick auf die politische Sprache und deren Bedeutungskontexte (Koselleck 2006, Steinmetz 2007). Die Annahme besagt, dass Begriffe kein bloßes Kommunikationsmittel sind, vielmehr formieren sie selbst Optionsmuster, nach denen Akteure ihr Handeln ausrichten. Begriffliche semantische Wandlungsprozesse liegen zumeist unter der öffentlichen Wahrnehmungsschwelle und werden erst durch empirische Rekonstruktion sichtbar. Bei dieser Rekonstruktion ist stets auch nach den jeweiligen ,Gegenbegriffen ' $\mathrm{zu}$ fragen (Koselleck 2006: 109-111). Gegenbegriffe erschöpfen sich nicht in lexikalischen ,Antonymen“ wie beispielsweise „arm“ versus „reich“, sondern bestimmen die impliziten Werte von Konzepten. Gegenbegriffe sind dabei häufig normativ asymmetrisch wie beispielsweise „Zivilisation“ versus „Barbarei“ oder „Kultur“ versus „Natur“ (ebd.: 170ff). Solche Unterscheidungen 
sind geprägt durch ihren spezifischen kulturhistorischen Kontext. Die folgende Analyse zeigt, dass der Gegenbegriff von „Sicherheitskultur“ „Schuldkultur“ lautet. Die „Schuldkultur" ist noch von einer asymmetrischen Anthropologie geprägt, die auf Sanktionsdrohungen gegen menschliche Akteure setzt, um Gefahren zu verringern. Demgegenüber richtet die Sicherheitskultur ihren Blick auf die Beziehungen zwischen Menschen, Dingen und Regeln.

\section{Quantifizierende Dimensionen}

Die Analyse stützt sich auf eine Quellenauswahl, die explizite Verwendungen des Begriffs „Sicherheitskultur“ in ausgewählten Tages- und Wochenzeitungen enthalten. ${ }^{2}$ Es wäre sicherlich wünschenswert weitere Synonyme zu berücksichtigen, dies würde jedoch den Arbeitsumfang überschreiten. Zudem vergrößert sich bei einer Ausweitung auf ähnliche Begriffe stets die Gefahr einer willkürlichen Auswahl des Quellenmaterials, da nie exakt die Grenze der Ähnlichkeiten bestimmbar ist. ${ }^{3}$ Die Kodierungen der Artikel erfolgten nach der klassischen Methode der Grounded Theory (Barney/Strauss 1967) und beschränkten sich auf die verschiedenen Bedeutungsgehalte des Begriffs selbst. Weitere ähnliche Begriffe wurden nicht be-

Tabelle 1. Themenkontext von „Sicherheitskultur“ in deutschen Printmedien $\left(\mathrm{n}=151^{4}\right)$

\begin{tabular}{|l|c|}
\hline Referenzdimensionen & $\begin{array}{c}\text { (n=Anzahl } \\
\text { Artikel })\end{array}$ \\
\hline Atomenergie & 99 \\
\hline $\begin{array}{l}\text { Umweltgefahren der } \\
\text { Öl- und Chemieindustrie }\end{array}$ & 12 \\
\hline Luftfahrt & 9 \\
\hline Gesundheitswesen & 9 \\
\hline Verteidigungsbündnisse & 7 \\
\hline Bahn & 3 \\
\hline Bau, Bergwerke & 3 \\
\hline Cyber, IT, Soziale Netzwerke & 2 \\
\hline Governance, Öffentlichkeit & 2 \\
\hline Automobil & 2 \\
\hline EU-Wirtschaftsraum & 1 \\
\hline Kriminalität & 1 \\
\hline RAF/Stammheim & 1 \\
\hline Extreme Wetterereignisse & 1 \\
\hline
\end{tabular}

2 Ausgewählt wurden die Tageszeitungen ,FAZ` und ,taz‘ sowie die Wochenzeitungen ,DIE ZEIT' und ,DER SPIEGEL'. Die Auswahl erfolgte über Datenbanken, die im Falle des SPIEGELS, DER ZEIT und der FAZ bis Ende der 1940er Jahre zurückreichen und in denen der Suchbegriff ,Sicherheitskultur*‘ wörtlich verwendet wird. Die Datenbank der taz reicht bis in das Jahr $1986 \mathrm{zu}-$ rück. Die Suchstrategie bezog sich auf die gesamten zur Verfügung stehenden Zeiträume.

3 Laut Koselleck (2006) sollen nur in begründeten Ausnahmefällen semantisch ähnliche Begriffe partiell berücksichtigt werden. In der Inhaltsanalyse nennt sich ein solches Vorgehen auch Kodierung erster Ordnung.

4 Quellen: FAZ, SPIEGEL, ZEIT, taz. Einige Texte aus dem Jahr 2010-11 wurden aus inhaltlichen Gründen mit aufgeführt (Tab. 1), obwohl sie in den diachronischen Vergleich (Tab. 2) aus systematischen Gründen nicht mit einbezogen wurden. rücksichtigt. Eine solche Beschränkung soll auch sicherstellen, dass ausreichend Raum für eine qualitative Tiefeninterpretation bleibt. ${ }^{5}$

Der Begriff „Sicherheitskultur“ findet sich seit seiner ersten Nennung im Jahre 1986 bis zum Ende des Jahres 2009 in 147 Artikeln der ausgewählten Zeitungen. Zu etwa zwei Drittel berichten die Artikel von der Sicherheitskultur der Atomindustrie (Tab. 1). Die Themen reichen von Störfällen in Reaktoren über Atomtransporte bis zu Sicherheitsfragen von Atommülllagern. Alle anderen Themen verteilen sich auf das restliche Drittel. Darunter fanden sich Fragen zu Umweltgefahren, die durch die Öl- und Chemieindustrie hervorgerufen werden, sowie zur Sicherheit in der Bauindustrie und im Bergbau. Des Weiteren wird die Sicherheitskultur von Fluggesellschaften, insbesondere im Zusammenhang mit Flugzeugabstürzen, diskutiert. Andere Artikel befassen sich mit dem Gesundheitswesen und den dortigen sicherheitskulturellen Mängeln wie Kunstfehler durch das medizinische Personal. Schließlich beschreiben die Medien Sicherheitskultur auch im Kontext von militärischen Bündnissen wie der NATO und der Nahostregion. Weitere Themen sind Internet und soziale Netzwerke, Automobilindustrie, EU-Finanzraum, Kriminalität und extreme Wetterlagen. In einem Fall wird sogar die Sicherheitskultur der Bundesrepublik in den Zusammenhang der RAF und der fehlenden Transparenz einer sicherheitskulturell geprägten öffentlichen Governance gestellt.

Chronologisch findet sich der Begriff erstmals am 17.09.1986 in der links-alternativen Tageszeitung taz. Thema des entsprechenden Artikels waren die Störungen in den Atomreaktoren in Cattenom (Frankreich) und Tschernobyl (ehem. Sowjetunion). Andere Zeitungen und Magazine wie die FAZ, der SPIEGEL und die ZEIT, greifen ihn erstmals im Jahr 1988 auf. Anlass war die Initiative des damaligen Umweltministers Klaus Töpfer (CDU), eine neue „Sicherheitskultur in der Industriegesellschaft“ (FAZ 29.06.1989) zu verwirklichen: Ziel dieser Sicherheitskultur sollte es sein „den Umweltschutz nicht zu einem Hemmschuh für den technologischen Fortschritt werden zu lassen " (FAZ 01.06.1989). Seither steigt die Zahl der Verwendungen stetig an. In der zweiten Hälfte der 2000er Jahre verdoppelte sich die Frequenz sogar (Tab. 2). Diese Zunahme ist einer Reihe von Störfällen in der Atomindustrie geschuldet, aber auch der inhaltlichen Ausdifferenzierung des Begriffs in andere Kontexte, vor allem in die Medizin.
5 Zur Methodik vgl. ausführlicher Rauer (2008). In Bezug auf die Geschichte der ,Security Studies' schlägt Ole Weaver (2011: 655) vor, „Medien“, „Policy-Makers“, „Stiftungen“, akademische „Netzwerke“ und „Hierarchien“ zu analysieren. Eine solche akteurszentrierte Analyse vernachlässigt jedoch die für eine Konzeptgeschichte unerlässliche Perspektive auf kulturelle Bedeutungssysteme. 
Tabelle 2. „Sicherheitskultur“ in den ausgewählten deutschen Printmedien gesamt (n=147*)

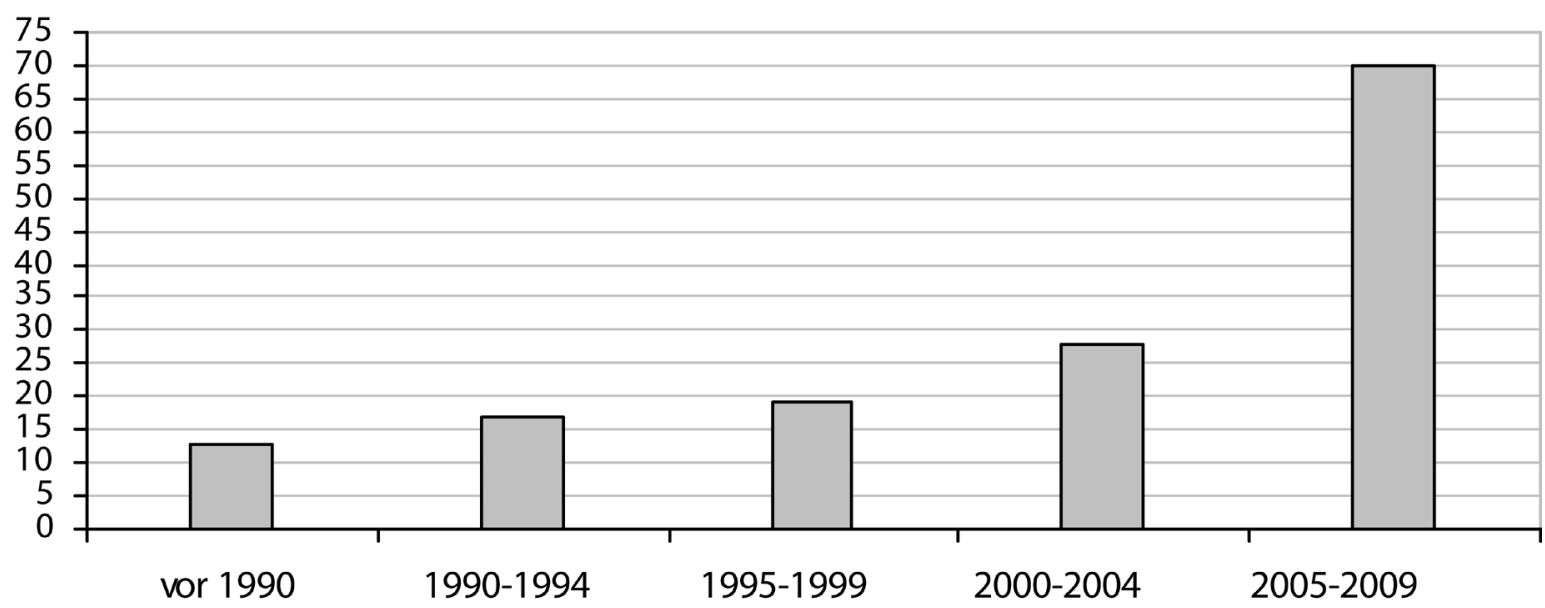

* $\quad \mathrm{N}$ = Nennung per Artikel (intra-textuelle Mehrfachnennungen nicht berücksichtigt); Quellen: FAZ, SPIEGEL, ZEIT, taz. Die Texte aus dem Jahr 2010-11 wurden in den diachronischen Vergleich aus systematischen Gründen nicht mit einbezogen.

Offenbar sind rein inhaltlich betrachtet dem Referenzhorizont von ,Sicherheitskultur‘ insgesamt keine Grenzen gesetzt. Zwar findet sich quantitativ eine gleich bleibendstarke Zentrierung auf Atomunfälle. Zu diesem Kernthema addieren sich aber stets neue Themenfelder hinzu. Die Referenzdimension hat sich also im Laufe der untersuchten zwanzig Jahre kontinuierlich erweitert. Damit steht die öffentliche Verwendungsweise von Sicherheitskultur im Einklang mit einem allgemeinen inhaltlichen Entgrenzungsprozess sicherheitspolitischen Denkens, dessen Geschichte sich bis zu den Anfängen des letzten Jahrhunderts zurückverfolgen lässt (vgl. Kaufmann 1973, Bonß 1995, Zwierlein/Graf/Ressel 2010, Daase 2010).

\section{Komparative und operative Funktionen von Sicherheitskultur}

Von der rein inhaltlichen Ebene lässt sich die semantische Funktion des Konzepts unterscheiden. Hier beschränkt sich das Konzept auf zwei Funktionen, die einer ausführlicheren Erläuterung bedürfen: eine komparative (ad. 1) und eine operative Funktion (ad. 2).

\section{Die komparative Funktion}

Der Begriff wird häufig dazu verwendet, die ,Sicherheitskultur ‘ verschiedener Gruppen wie beispielsweise von Unternehmen, Nationen oder politischen Systemen, aber auch von Kontinenten wie ,Europa' und ,Amerika' zu vergleichen. Zumeist geschieht dies anhand der Unterscheidung ,besser'/'schlechter'. So schreibt der SPIEGEL im Jahre 1988: „Östliche Schlampereien seien in der deutschen Sicherheitskultur undenkbar“ (SPIEGEL 11.01.1988). Im Laufe der Jahre wandeln sich dabei lediglich die adressierten Kollektive, die vergleichende Semantik bleibt die Gleiche, wie beispielsweise in diesem Zitat der FAZ: „So sagte der Vorsitzende des Staatlichen Komitees der Ukraine für Kernsicherheit, Nicolai Schtejnberg [...]: ,In einem totalitären Staat könne es keine Sicherheitskultur geben, da zu ihren wesentlichen Grundlagen das Zweifeln und das Recht auf freie Meinungsäußerung gehörten““ (FAZ 11.12.1991). Neben politischen und kontinentalen Aggregierungen finden sich stets auch die klassisch nationalen Vergleiche. Beispielsweise schreibt die FAZ mit Blick auf eine gemeinsame Sicherheitskultur der Europäischen Union (EU) in technischen und wirtschaftlichen Belangen: „Im Verlaufe ihrer industriellen Entwicklung sind in Großbritannien, Frankreich und Deutschland unterschiedliche Sicherheitskulturen entstanden. Diese Systeme der ,Produktion von Sicherheit' sind ebenso komplex wie die Anlagen, deren Sicherheit garantiert werden soll. Es macht keinen Sinn, ein solches System zu exportieren, und wer es mit Elementen eines anderen verschneidet, geht erhebliche Risiken ein“ (FAZ: 22.09.1992).

Wie diese drei Beispiele demonstrieren, ist die komparative Funktion dem zweiten Begriffsteil - der ,Kultur‘ - geschuldet. Dieses komparative Verständnis von ,Kultur' setzt voraus, dass sich Einheiten klar und präzise unterscheiden lassen und homogen für eine ganze Nation oder ein politisches System gelten. Andreas Reckwitz nennt ein solches, sich auf ein gesamtes Kollektiv erstreckendes Kulturverständnis „holistisch“ “6 Was sich unterscheidet, sind nicht die objektiven Sicherheitslagen selbst, sondern die kollektiv geteilten Interpretationsweisen und Bewertungen von etwas als „bedrohlich“ oder „sicher“. Damit hat der Kulturaspekt am Konzept der Sicherheitskultur auch eine relativierende und subjektivierende Funktion. Wenn unterschiedliche Akteure Sicherheit und Bedrohung unterschiedlich interpretieren, so handelt es sich nicht mehr um rein objektivierbare Bedrohungen, sondern um relative Sichtweisen aufgrund von tradierten Erfahrungen und kollektiver Gewohnheiten.

6 Der Kultursoziologe Andreas Reckwitz (2006; 2010) unterscheidet vier Bedeutungsdimensionen von „Kultur“: Erstens, Kultur als Wert im Sinne von Sitten, Tugenden und Moralvorstellungen (nach Immanuel Kant), zweitens, Kultur als Totalität von Gesellschaften, Ethnien oder Nationen (nach Gottfried Herder), drittens, Kultur als ein Funktionssystem für Literatur, Kunst und Theater (nach Talcott Parsons) sowie viertens, Kultur als Bedeutungsnetz zur Interpretation von sozialen Situationen, Handlungen und Akteuren (nach Clifford Geertz). 
Ein solches holistisches Kulturverständnis kann jedoch nicht nur relativierend, sondern auch kontrastierend interpretiert werden. Bekanntermaßen führte Tschernobyl schlagartig vor Augen, dass das Denken in Kategorien nationaler Sicherheit keinen Schutz vor Katastrophen garantiert. Die Referenz auf ,Kultur' reagiert auf diese neue Situation. Die Unterscheidung einer sicherheitskulturellen Differenz dient einer Grenzziehung zwischen dem ,sicheren Wir‘ und dem ,unsicheren Anderen‘. ,Unsere‘ Sicherheitskultur ist vertrauenswürdiger als die ,der Sowjets“ oder ,der Franzosen'. Der Kulturbegriff rekonstruiert eine verloren gegangene nationale Sicherheitsvorstellung. Aus Sicht der vermeintlich fortschrittlicheren nationalen Sicherheitskultur hat diese komparative Funktion offenbar den Nebeneffekt, das subjektive Sicherheitsempfinden zu fördern und die eigenen Ängste vor den Technologien zu lindern. Ein solches wechselseitiges Bedingungsverhältnis wurde in der Sicherheitsforschung vielfach beschrieben (Kaufmann 1973: 297f; Porzsolt 2007; Biess 2009, Arndt 2010).

Gleichwohl handelt es sich bei der komparativen Semantik nicht immer um einen pauschalisierenden sicherheitsspezifischen Multikulturalismus. Denn oftmals kombinieren die Vergleiche Pauschalisierungen mit objektivierbaren institutionellen Praktiken, wie in diesem Beispiel aus der FAZ zur der Sicherheitskultur von Kernkraftwerken: „,Deutschland hat nachweislich die höchste Sicherheitskultur،, versichert Harig. Die Energieversorger könnten den Betrieb nur verantworten, weil die Kernkraftwerke so ausgelegt seien, daß Fehler toleriert werden könnten." (FAZ 13.06.1998) Und in einem europäischamerikanischen Vergleich der Sicherheitskulturen zitiert die FAZ einen Experten der Bundesanstalt für Geowissenschaften: „Am meisten hapere es in Amerika an der Kontrolle: ,Dort ist die Genehmigungsbehörde zugleich die Kontrollbehörde, und sie kassiert auch für die Lizenzen. ' Nach seiner Ansicht muss das Personal besser geschult und für Risiken sensibilisiert werden“ (FAZ 06.06.2010). Beide Zitate koppeln den Vergleich von kulturellen Einheiten an die Differenz institutioneller Praktiken. Die komparative Funktion adressiert also nicht nur kollektive Einheiten, sondern auch institutionelle Praktiken.

\section{Die operative Funktion}

Die zweite semantische Funktion von Sicherheitskultur folgt nicht einer vergleichenden, sondern einer operativen Semantik. ${ }^{7}$ Um die operative Begriffsfunktion zu erfassen, ist es aufschlussreich den ursprünglichen Entstehungszusammenhang des Konzepts zu rekonstruieren. Der Reaktorunfall in Tschernobyl hatte offenbar eine epistemologische Lücke hinterlassen. Die verfügbaren Begriffe wie Risikoabschätzung, Gefahrenbewusstsein, Bedienungsfehler oder menschliches Versagen reichten nicht aus, um die Unfallsursachen adäquat zu erklären. Dies geht aus dem Dokument der Sondersitzung der internationalen Atomenergiebehörde (IAEA) hervor. Dort heißt es, dass mit Tschernobyl „ein Ereignis Wirklichkeit geworden ist, von dem man glaubte, dass es eine extrem niedrige Eintrittswahrscheinlichkeit habe“ (INSAG I: 1986: 98). Risikokalkulati-

7 Diese operative Semantik von Sicherheitskultur ähnelt dem, was bisweilen auch „Sicherheitsmanagement" genannt wird (Weick 1995, Guldemund 2010). Dabei bleibt jedoch die vergleichende Funktion unberücksichtigt. onen erwiesen sich für eine Ursachenanalyse als unbrauchbar. In der Zusammenfassung stellt der Bericht fest: „Es lassen sich keine physikalischen Phänomene feststellen, die nicht bereits zuvor in Sicherheitsanalysen [...] und/oder [der] experimentellen Forschung erkannt worden wären“ (INSAG I: 1986: 108). Tschernobyl wurde nicht durch technologische Wissensdefizite verursacht. Technisch und physikalisch war vorher bekannt, dass eine solche Bedienung des Reaktors fatale Konsequenzen haben würde. In den Termini von Sicherheit und Wissen bezog sich diese Problematik also nicht auf technologische Unwahrscheinlichkeiten, den „Known Unknowns“ oder gar völligen Ungewissheiten, den „Unknown Unknowns“ (Daase/Kessler 2007), sondern auf die „Knowns“ - also auf vorhandenes bekanntes Wissen. Die Tatsache, dass das technologische Wissen vorhanden war und sich der Unfall dennoch ereignete, offenbarte die epistemologische Leerstelle. Der Begriff „Sicherheitskultur" wurde eingeführt, um diese Lücke zu schließen.

In dem ersten Bericht der INSAG I erfolgte die Einführung ohne eine explizite Definition. ${ }^{8}$ Lediglich vage wurden unter Sicherheitskultur drei Dimensionen des „sogenannten ,menschlichen Elements'“ (INSAG I 1986: 108) subsumiert: Erstens, die Förderung eines tieferen Grundverständnisses des Bedienungspersonals durch den „Einsatz von Simulatoren“ (ebd.). Zweitens, interne und externe Kontrolle um „Leichtfertigkeit zu verhindern, die sich aus dem Routinebetrieb ergeben könnte“ (ebd.). Drittens, Stärkung des „ständigen Bewusstseins seitens des gesamten Personals“ für die „Auswirkungen auf die Sicherheit im Fall einer Abweichung von den Vorschriften“ (ebd.). Wie diese vage Liste zur näheren Qualifizierung von Sicherheitskultur zeigt, zeugte Tschernobyl von einem „kulturelleren“ Problem, das nach Optimierung der sozialen Abläufe und Interaktionsformen verlangt. Der Bericht fordert erstens Übungen an Simulatoren, die ein „praxeologisches Wissen“ (Reckwitz 2010) verbessern sollen. Zweitens werden die Gefahren genannt, die aus einem Zuviel an praxeologischem Wissen - aus Routinen - resultieren. Schließlich wird drittens keine Optimierung des Risikobewusstseins gefordert, sondern die Optimierung des Bewusstseins über die sicherheitstechnischen Konsequenzen von Regelbrüchen. Nicht ein strikteres Verbot von Regelverstößen bietet die Lösung, sondern das präzisere Bewusstsein um die Folgen von Regelverstößen. Regelverstöße gelten in spezifischen Situationen als rettende Maßnahme des letzten Augenblicks, sie dürfen niemals a priori verboten werden (Ortmann 2003). Zu Optimieren ist also das Bewusststein vom ,richtigen' Verhältnis von Regel und Ausnahme.

In den folgenden Abschnitten betont der Bericht die „Bedeutung einer befriedigenden Mensch-Maschine-Wechselwirkung“ (INSAG I: 1986: 110). Die Sicherheitskultur des Reaktors in Tschernobyl stamme aus einer Zeit, die „menschliche Eingriffe in das Schutzsystem auch zur unsicheren Seite hin erlaubte. Seinerzeit glaubte man, dass der Mensch zuverlässiger sei als die verfügbaren technischen Systeme“ (ebd.: 5). Diese Ursachenzurechnung des Berichts ist bemerkenswert, wird doch behauptet, dass die Sicherheitskultur von Tschernobyl von einer, wie Bruno Latour (1998) es nennen würde, asym-

8 Diese erfolgte erst in einem späteren Dokument aus dem Jahre 1991, vgl. dazu ausführlich: Büttner/Fahlbruch/Wilpert (1999: 45-50). 
metrischen Anthropologie der Moderne geprägt war. In Science and Technology Studies wird die asymmetrische Anthropologie dafür kritisiert, dass sie den Menschen über oder unter den Dingen verorte: Entweder beherrsche der Mensch die Technik oder die Technik beherrsche den Menschen. Diese asymmetrische Anthropologie verkennt, dass menschliche Akteure mit technischen Aktanten ein komplexes Netzwerk von Schnittstellen bilden, in der keine Seite strukturell überlegen ist (Latour 1998, Nowottny 2005, Guggenheim/Potthast 2011). Das Sicherheitskulturkonzept aus dem Bericht INSAG I könnte vor diesem Hintergrund als früher epistemischer Versuch einer ReSymmetrisierung des Missverhältnisses zwischen Mensch und Technik gedeutet werden.

In der Öffentlichkeit wurde dieses Begriffsverständnis nur wenige Monate nach Tschernobyl von der taz, einen französischen Experten zitierend, implizit verwendet: „François Cogne sprach von den französischen AKWs als von den, sichersten der Welt'. Anders als bei Tschernobyl hätten die Franzosen die ,Schnittstelle Mensch-Maschine‘ durch bessere Ausbildung und Schulung des Personals im Griff. ,Technisch gesehen“ bestünde ,keinerlei Anlass für Veränderungen' in der französischen Atomtechnik. Man habe eine ,echte Sicherheitskultur ${ }^{\star}$ entwickelt. Einen Unfall könne zwar niemand ausschließen, aber Cogne sieht keinerlei ,gravierenden Unfall, der die Umgebung in Mitleidenschaft ziehen könnte‘." (taz 17.09.1986) Diese operative Funktion hat sich in der publizistischen Öffentlichkeit bis heute verstetigt. Der Verweis auf Sicherheitskultur bezieht sich zumeist auf die Beschaffenheit de der Schnittstelle zwischen Menschen, Dingen und Regeln. So heißt es beispielsweise im Jahre 2002 in der ZEIT: „Einigkeit besteht aber über Folgendes: Um die Risiken der Atomenergie zu minimieren, ist eine hoch entwickelte Sicherheitskultur erforderlich, sowohl bezüglich der technischen Rahmenbedingungen als auch bezüglich der politischen, organisatorischen und gesellschaftlichen Umstände.“ (ZEIT: 25.07.2002) Es handelt sich bei der operativen Semantik also nicht mehr um einen holistischen Kulturbegriff, sondern um einen bedeutungsorientierten Kulturbegriff (Reckwitz 2010; Daase in diesem Heft).

Die operative Semantik findet sich auch in den weiteren thematischen Feldern wie der Öl- und Chemieindustrie, der Bahnund Automobilwirtschaft und dem Militär. Besonders ausgeprägt ist sie jedoch in der Medizin. So schreibt die FAZ im Jahre 2000 unter dem Titel: „Gesundheitswesen ohne Sicherheitskultur“: „Die Fehlerrate im Gesundheitswesen ist unzumutbar hoch'. [...] Das Ziel ist ehrgeizig, denn es geht darum, einem ganzen Industriezweig - dem Gesundheitswesen - zu einer modernen Sicherheitskultur zu verhelfen" (FAZ 05.01.2000). Anlass war ein Bericht, den das Institut für Medizin der U.S.amerikanischen Nationalen Akademie der Wissenschaften unter dem Titel „To Err Is Human. Building a Safer Health System“ veröffentlicht hatte (Kohn/Corrigan/Donaldson 2000). Im Bericht wurde kritisiert, dass es zu wenig Kooperation zwischen dem Büro für Zulassung von Arzneimitteln und dem Büro für Überwachung und Epidemiologie bereits zugelassener Medikamente gäbe. Eine neue Sicherheitskultur entwickelt sich seither, bei der die Risiken und Nutzen der Medikamente „über den ganzen Lebenszyklus eines Präparats verfolgt werden“ (FAZ 20.12.2006). Im Zuge dieser medizinischen Sicherheitskultur wurden von den deutschen Institutionen des Gesundheitswesens Programme entwickelt, wie beispielsweise die Initiative „Aktionsbündnis Patientensicherheit“. ${ }^{9}$ Neben der Durchführung zahlreicher Informations- und Fortbildungsmaßnahmen organisiert die Initiative eine Plattform, die dazu dient die Häufigkeit von Behandlungsfehlern dadurch zu senken, dass die Fehler anonym gemeldet werden können. Diese Meldungen bilden die Vorraussetzung für Verbesserungsmaßnahmen. Andere fordern Praktiken des Innehaltens, die sogenannten „team-time-outs“ (FAZ 19.09.2007). Dabei handelt es sich um institutionalisierte Pausen im operativen Ablauf, in denen letzte Zweifel vor dem Beginn einer Operation nochmals kommuniziert werden müssen. Bei all diesen Vorschlägen geht es also auch um eine Optimierung der Relation zwischen Mensch-Maschine und Mensch-Mensch.

Diese Initiativen werden in der medizinischen Fachliteratur auch mit der Formel: „Von der ,culture of blame“ zur Sicherheitskultur" (Hempelmann/Quinzio 2000: 421) zusammengefasst. Die Schuldkultur sucht den operativen Fehler allein bei den Menschen und setzt auf Sanktionen. Damit verhindert sie die Meldung unentdeckter Fehler und die Möglichkeit sie in Zukunft zu vermeiden. Schuldkultur setzt auf die Bestrafung und das Primat des Menschen über die Dinge und folgt den Annahmen einer asymmetrischen Anthropologie. Sicherheitskultur sieht im Gegensatz dazu in den menschlichen Akteuren nicht mehr das Primat und die Hauptverantwortung aller sicherheitsrelevanten Faktoren. Dies bedeutet selbstverständlich nicht, dass jede persönliche Verantwortung obsolet werde, es bedeutet lediglich, dass der Appell an das persönliche Verantwortungsbewusstsein nicht mehr ausreicht, um Sicherheit komplexer gesellschaftlich-technologischer Interaktionen zu gewährleisten. Letzteres haben insbesondere Franz Xaver Kaufmann (1973) und Charles Perrow (1989) ausgearbeitet, ohne jedoch die semantischen Schlussfolgerungen daraus zu ziehen. Ein Konzept von „Kultur“ fehlt in beiden Werken vollständig. Ähnliches gilt für die Risikosoziologie. Der Risikobegriff (Beck 1986) richtete sich noch an der asymmetrischen Anthropologie aus. Risiko erhebt das rationale Kalkül der Akteure zum Primat der Analyse. Der kulturorientierte Sicherheitsbegriff geht hingegen von symmetrischen Wechselwirkungen aus. Insgesamt zeigt die Analyse also, dass sich die Semantik der Sicherheitskultur weder ausschließlich mit menschlichen Akteuren noch technischen Systemen befasst. Vielmehr artikuliert sie vernetzte Zusammenhänge zwischen kulturellen Deutungsmustern, institutionalisierten Praktiken und technischen Systemen, ohne einem der Elemente eine Priorität zuzurechnen.

\section{Fazit}

Seit dem Reaktorunfall von Tschernobyl im Jahre 1986 wurde der Begriff Sicherheitskultur in deutschen Printmedien verwendet. Das Kernthema des Konzepts ist die Reaktorsicherheit. Daneben differenziert sich der Begriff quantitativ und inhaltlich kontinuierlich weiter aus. Neben der stetigen Verwendung in der Atomindustrie findet er sich beispielsweise auch in verteidi-

9 Vgl. für Hinweise und Downloads: http://www.aktionsbuendnis-patientensicherheit.de/. 
gungspolitischen Zusammenhängen, z.B. in Texten der NATO oder zur Beschreibung des Umgangs mit Kriminalität in Rio de Janeiro oder Medikamenten und Hygiene in Krankenhäusern.

Auf der semantischen Ebene lassen sich zwei unterschiedliche Verwendungsweisen des Sicherheitskulturbegriffs feststellen: eine operative und eine komparative Funktion. Die komparative Funktion bezieht sich auf kollektive Akteure wie „Deutschland“ oder „Frankreich“, „Europa“ oder „Amerika“, „Westen“ oder „Osten“. Der Verweis auf Sicherheitskultur setzt in diesem Verwendungszusammenhang die Einheit eines Kollektivs voraus, um es mit einer anderen Einheit mit Blick auf Ähnlichkeiten oder Differenzen zu kontrastieren. Die komparative Semantik relativiert und subjektiviert den Umgang mit Sicherheit. Der kulturelle Vergleich stiftet selbst immer dann Sicherheit, wenn die Sicherheitspraktiken der ,Anderen' als weniger effektiv qualifiziert werden. Damit hat er eine implizite re-kollektivierende Funktion in Zeiten globaler Entgrenzungen.

Die zweite wesentliche Funktion des Konzepts ist die operative Semantik, die nicht auf kollektive Einheiten zielt, sondern auf Relationen zwischen Mensch-Maschine, Mensch-Mensch und Mensch-Regel. Hier folgt das Konzept implizit der Vorstellung einer symmetrischen Anthropologie, in der die Zuverlässigkeit des Menschen nicht länger über die Zuverlässigkeit der Dinge gestellt wird. Semantisch lautet der Gegenbegriff zu Sicherheitskultur also nicht „Fahrlässigkeit“ und „Verantwortungslosigkeit“, sondern „Schuldkultur“. Wenn die Medien Sicherheitskulturen beobachten, dann blicken sie weniger auf das Risiko- und Fehlverhalten von rationalen Akteuren, sondern auf soziale Relationen und kulturelle Bedeutungssysteme.

\section{Literatur}

Arndt, Melanie 2010: From Nuclear to Human Security? Prerequisites and Motives for the German Chernobyl Commitment in Belarus, in: Historical Social Research, 35: 4, 289-308.

Beck, Ulrich 1986: Risikogesellschaft. Auf dem Weg in eine andere Moderne, Frankfurt/M.

Biess, Frank 2009: 'Everybody Has a Chance'. Civil Defense, Nuclear Angst, and the History of Emotions in Postwar Germany, in: German History 27: 2, 215-243.

Bonß, Wolfgang 1995: Vom Risiko: Unsicherheit und Ungewissheit in der Moderne. Hamburg.

Büttner, Torsten/Fahlbruch, Babette/Wilpert, Bernhard 1999: Sicherheitskultur: Konzepte und Analysemethoden, Heidelberg.

Conze, Eckart 2005: Sicherheit als Kultur. Überlegungen zu einer „modernen Politikgeschichte“ der Bundesrepublik Deutschland, in: Vierteljahrshefte für Zeitgeschichte 53, 357-380.

Conze, Werner 1984: Sicherheit, Schutz. In: Brunner, Otto/Conze, Werner/Koselleck, Reinhart (Hrsg.): Geschichtliche Grundbegriffe. Historisches Lexikon zur politisch-sozialen Sprache in Deutschland, Stuttgart, 831-862.
Daase, Christopher/Kessler, Oliver 2007: Knowns and Unknowns in the 'War on Terror': Uncertainty and the Political Construction of Danger, in: Security Dialogue, 38: 4, 411-436.

Daase, Christopher 2010: National, Societal, and Human Security: On the Transformation of Political Language, in: Historical Social Research, 35: 4, 22-37.

Glaser, Barney G./Strauss, Anselm 1967: The Discovery of Grounded Theory. Strategies for Qualitative Research, Chicago.

Guggenheim, Michael/Potthast, Jörg, i.E., 2011: Symmetrical Twins. On the Relationship Between Actor-Network-Theory and the Sociology of Critical Capacities. In print: European Journal of Sociological Theory [als Pdf-Version im Internet verfügbar].

Guldenmund, Frank W. 2010: (Mis)understanding Safety Culture and Its Relationship to Safety Management, in: Risk Analysis, 30: 10, 1466-1480.

Härter, Karl 2010: Security and "gute Policey" in Early Modern Europe: Concepts, Laws and Instruments, in: Historical Social Research, 35: 4, 41-65

Hempelmann, Gunter/Quinzio, Lorenzo 2000: Sicherheit in der Anästhesie - von der „culture of blame“ zur Sicherheitskultur, in: Gunter Hempelmann (Hrsg.): Anästhesiologie, Intensivmedizin, Notfallmedizin, Schmerztherapie, Stuttgart, 421-422.

INSAG I 1986: Maßnahmen zur Stärkung der internationalen Zusammenarbeit auf dem Gebiet der Reaktorsicherheit und des Strahlenschutzes: Sondersitzung der Generalkonferenz der Internationalen Atomenergiebehörde vom 24. bis 26. September 1986 in Wien. Teil: 1. Zusammenfassender Bericht einschließlich der deutschen Übersetzung des INSAG Berichtes über die Analyse des Unfalls im Kernkraftwerk Tschernobyl; Körperschaft: International Atomic Energy Agency; Hrsg. vom Bundesminister für Umwelt, Naturschutz und Reaktorsicherheit, Bonn, Referat RS 18, 1986.

Kaufmann, Franz-Xaver 1973: Sicherheit als soziologisches und sozialpolitisches Problem: Untersuchungen zu einer Wertidee hochdifferenzierter Gesellschaften, Stuttgart.

Koselleck, Reinhart 2006: Begriffsgeschichten. Studien zur Semantik und Pragmatik der politischen und sozialen Sprache, Frankfurt/M.

Kohn, Linda T./Corrigan, Janet M./Donaldson, Molla S. (Eds.) 2000: To Err Is Human. Building a Safer Health System. Committee on Quality of Health Care in America. Institute of Medicine, Washington, D.C.

Latour, Bruno 1998: Wir sind nie modern gewesen. Versuch einer symmetrischen Anthropologie, Frankfurt/M.

Nowottny, Helga 2005: The increase of complexity and its reduction: Emergent Interfaces Between the Natural Sciences, Humanities and Social Sciences, in: Theory Culture \& Society, 22: $5,15-31$.

Ortmann, Günther 2003: Regel und Ausnahme. Paradoxien sozialer Ordnung, Frankfurt/M.

Perrow, Charles 1989: Normale Katastrophen: die unvermeidbaren Risiken der Großtechnik, Frankfurt/M. 
Porzsolt, Franz 2007: Gefühlte Sicherheit - Ein Entscheidungskriterium für Patienten? In: Zeitschrift für Allgemeinmedizin, 83, 501-506.

Rauer, Valentin 2008: Die öffentliche Dimension der Integration. Migrationspolitische Diskurse türkischer Dachverbände in Deutschland, Bielefeld.

Reckwitz, Andreas 2010: Kultursoziologische Analytik zwischen Praxeologie und Poststrukturalismus, in: Monika Wohlrab-Sahr (Hg.): Kultursoziologie. Paradigmen - Methoden - Fragestellungen, Wiesbaden, 179- 205.

Reckwitz, Andreas 2006: Die Transformation der Kulturtheorien. Zur Entwicklung eines Theorieprogramms. Mit einem Nachwort zur Studienausgabe 2006: Aktuelle Tendenzen der Kulturtheorien, Weilerswist.
Schenk, Gerrit Jasper 2010: ,Human Security“ in the Renaissance? Securitas, Infrastructure, Collective Goods and Natural Hazards in Tuscany and the Upper Rhine Valley, in: Historical Social Research, 35: 4, 209-233.

Steinmetz, Willibald 2007: Neue Wege einer historischen Semantik des Politischen, in: Willibald Steinmetz (Hg.), ,Politik'. Situationen eines Wortgebrauchs im Europa der Neuzeit, Frankfurt/M, 9-40.

Woever, Ole 2010: Towards a Political Sociology of Security Studies, in: Security Dialogue, December, 22, 41: 6, 649-658.

Weick, Karl E. 1995: Der Prozess des Organisierens, Frankfurt/ M.

Zwierlein, Cornel/ Graf, Rüdiger/ Ressel, Magnus (Eds.) 2010: The Production of Human Security in Premodern and Contemporary History, Historical Social Research, HSR, 35: 4, Special Issue: Human Security.

\title{
Die neue Ambivalenz in der Sicherheitspolitik: Sicher- heitskultur als tiefer Kontext
}

\author{
Georgios Kolliarakis*
}

\begin{abstract}
With regard to contemporary security threats that may severely disrupt social normality, policies for civil security meet the limits of rational calculability and planning. Civil security is increasingly defined in reference to the vulnerability of critical infrastructure, and this has given rise to distinct counterterrorism policy patterns, such as focusing on low-probability high-impact events, resorting to symbolic politics, and favouring high-tech solutions. Prioritizing new issues as security threats and rendering certain policy options as adequate solutions are manifestations of security culture. Security culture provides a deep context for policy-making and helps reduce uncertainty and ambiguity in order to overcome political dilemmas and enable policy decisions. Still, security culture is an ambivalent mechanism that can enhance or undermine the ability of a society to respond to present problems and anticipate future ones.
\end{abstract}

Keywords: Civil security, uncertainty, security culture, policy patterns, ambivalence Zivile Sicherheit, Ungewissheit, Sicherheitskultur, Politikmuster, Ambivalenz

\section{Einleitung}

A usgangspunkt für diesen Beitrag ist die Beobachtung, dass sich westliche Gesellschaften heute neuartigen Bedrohungslagen ausgesetzt sehen, die eine besondere Herausforderung für deren zivile Sicherheit darstellen. Neben konkurrierenden gesellschaftlichen Interessen bei der Priorisierung dieser wahrgenommenen Bedrohungen und finanziellen und technologischen Möglichkeiten bei der Entwicklung und Implementierung passender Sicherheitsmaßnahmen, durch die Sicherheitspolitik

\footnotetext{
Georgios Kolliarakis ist wissenschaftlicher Mitarbeiter an der Professur für Internationale Organisationen im ExzellenzCluster „Die Herausbildung Normativer Ordnungen“ der Goethe-Universität Frankfurt a.M. Er möchte sich bei zwei anonymen Gutachtern bzw. Gutacherinnen und den S+F Redakteurinnen und Redakteuren für ihre hilfreichen Kommentare sowie bei Beate Stein und Martin Schmetz für die sprachliche Glättung des Textes herzlich bedanken.
}

beeinflusst wird, existiert ein weiterer Faktor, der meistens im Hintergrund seine Wirkung entfaltet: Sicherheitskultur. Sicherheitskultur „übersetzt“ einerseits bestimmte Phänomene selektiv in Bedrohungen und vermittelt andererseits bei sicherheitspolitischen Entscheidungs- und Handlungsabläufen. Sicherheitskultur lässt als tiefer Kontext der Politik Bedrohungsdiagnosen sowie Therapiemaßnahmen oft als selbstevident erscheinen. Gleichzeitig, indem oft auf eine bestimmte Palette von Maßnahmen und Prozessen fokussiert wird, werden notwendigerweise Alternativen an den Rand verschoben und kaum mehr wahrgenommen. In diesem Beitrag ersetzt Kultur weder als erklärende Variable die Rolle des rationalen Kalküls und der materiellen und rechtlichen Bedingungen bei der Gestaltung von Sicherheitspolitik, noch wird sie kausal durch die Politik beeinflusst. Sicherheitskultur wirkt eher als Kontextbedingung, sie liefert einen Deutungsrahmen, innerhalb dessen Bedrohungsdiagnosen 